\title{
Consideraciones sobre el estado de salud de adultos mayores durante la COVID-19
}

\section{Considerations on older adults health status during COVID-19}

Dayana Y. Garamende-Gutiérrez, Grecia M. Nolasco-Ramos y Luis A. Llanco-Albornoz*

Escuela Profesional de Medicina Humana, Universidad Privada San Juan Bautista, Ica, Chincha, Perú

Señor Editor,

Recientemente hemos leído el artículo de GuzmánOlea, et al. ${ }^{1}$, donde evaluaron integralmente a adultos mayores (AM) que padecieron enfermedad por coronavirus 2019 (COVID-19), sin embargo, nos hemos percatado de que dentro de dicha evaluación no se ha incluido un componente básico dentro del abordaje del paciente anciano, que es el deterioro cognitivo. La fragilidad en los AM aumenta el riesgo de infecciones al tiempo que disminuye la respuesta inmunitaria, lo que pone a esta población específica en mayor riesgo. Dantas-Araújo, et al. ${ }^{2}$ reportaron que entre las condiciones de riesgo de COVID-19 grave en los AM, el deterioro cognitivo es la más frecuente, pues está presente en el $57.5 \%$ impactando en la calidad de vida de estos. A su vez, Bayrak, et al. ${ }^{3}$ observaron que el deterioro cognitivo y la depresión incrementan en 15 veces más el riesgo de morir por COVID-19 en los AM. Infortunadamente, la pandemia continúa propagándose, afectando a más personas cada día; por ello, es necesario prestar atención en la evaluación integral del AM a fin de prevenir graves complicaciones, incluso la muerte.

\section{Financiamiento}

Los autores no recibieron patrocinio para el desarrollo de la presente carta al editor.

\section{Conflicto de intereses}

Los autores declaran no tener conflicto de intereses.

\section{Bibliografía}

1. Guzmán-Olea E, Agis-Juárez RA, Bermúdez-Morales VH, Torres-Poveda K, Madrid-Marina V, López-Romero D, et al. Estado de salud y valoración gerontológica en adultos mayores mexicanos ante la pandemia por COVID-19. Gac Med Mex. 2020;156(5):420-5.

2. Andrade FLJP de, Lima JMR de, Fidelis $\mathrm{K}$ do NM, Jerez-Roig J, Lima KC de. Cognitive impairment and associated factors among institutionalized elderly persons in Natal, Rio Grande do Norte, Brazil. Rev Bras Geriatr Gerontol. 2017;20(2):186-96.

3. Bayrak M, Çadirci K. The associations of life quality, depression, and cognitive impairment with mortality in older adults with COVID-19: a prospective, observational study. Acta Clin Belg. 2021;5:1-8.

\section{Correspondencia:}

*Luis A. Llanco-Albornoz

E-mail: luis.llanco@upsjb.edu.pe
Fecha de recepción: 17-05-2021

Fecha de aceptación: 20-05-2021

DOI: 10.24875/GMM.21000307
Gac Med Mex. 2021;157:571

Disponible en PubMed www.gacetamedicademexico.com 0016-3813/C 2021 Academia Nacional de Medicina de México, A.C. Publicado por Permanyer. Este es un artículo open access bajo la licencia CC BY-NC-ND (http://creativecommons.org/licenses/by-nc-nd/4.0/). 